\title{
Aplicações da cirurgia videolaparoscópica em pacientes pediátricos: uma revisão
}

\section{sistemática de literatura}

\author{
Applications of laparoscopic surgery in pediatric patients: a systematic literature review \\ Aplicaciones de la cirugía laparoscópica en pacientes pediátricos: revisión sistemática de la \\ literatura
}

Recebido: 11/11/2021 | Revisado: 19/11/2021 | Aceito: 19/11/2021 | Publicado: 28/11/2021

\author{
Bárbara Queiroz de Figueiredo \\ ORCID: https://orcid.org/0000-0003-1630-4597 \\ Centro Universitário de Patos de Minas, Brasil \\ E-mail: barbarafigueiredo@unipam.edu.br \\ Bárbara Andrade Cruz \\ ORCID: https://orcid.org/0000-0001-7802-3809 \\ Centro Universitário Euroamericano, Brasil \\ E-mail: barbara.andradecruz@ gmail.com \\ Janayna Fraga Teixeira \\ ORCID: https://orcid.org/0000-0001-8682-9394 \\ Centro Universitário de Goiatuba, Brasil \\ E-mail: janaynafragateixeira@gmail.com \\ Lorena Martins Nagata \\ ORCID: https://orcid.org/0000-0003-1487-6436 \\ Centro Universitário de Goiatuba, Brasil \\ E-mail: lorena-mvet@live.com \\ Lunalva Gabrielli Veras Sousa \\ ORCID: https://orcid.org/0000-0001-5181-5371 \\ Centro Universitário Uninovafapi, Brasil \\ E-mail: veraslunalva@gmail.com \\ Mauro Soares Marra \\ ORCID: https://orcid.org/0000-0002-0543-1569 \\ Centro Universitário Atenas, Brasil \\ E-mail: maurosoaresm@gmail.com \\ Edson Antonacci Júnior \\ ORCID: https://orcid.org/0000-0001-5118-4573 \\ Centro Universitário de Patos de Minas, Brasil \\ E-mail: edsonantonacci@unipam.edu.br
}

\begin{abstract}
Resumo
Introdução: a cirurgia laparoscópica é um dos cinco principais campos de progresso na cirurgia pediátrica na última década do século XX e tem sido um avanço progressivo o que permitiu o desenvolvimento de um grande número de procedimentos cirúrgicos por técnica minimamente invasiva. No entanto, cabe analisar de forma especial como essa técnica se desenvolve em pacientes pediátricos, visto que, há complicações e aplicações para esse grupo da população, que necessita de avaliações criteriosas quando se realiza procedimentos. Objetivo: analisar relatos de caso, artigos e estudos que mostrem quais são as vantagens e as desvantagens da realização da cirurgia videolaparoscopia em pacientes pediátricos Metodologia: Trata-se de uma revisão sistemática de literatura com estudos publicados nas seguintes bases de dados: National Library of Medicine (PubMed MEDLINE), Scientific Electronic Library Online (Scielo), Cochrane Database of Systematic Reviews (CDSR), Google Scholar, Biblioteca Virtual em Saúde (BVS) e EBSCO Information Services. Foram analisadas fontes relevantes inerentes ao tema, utilizando como um dos principais critérios a escolha de artigos atuais, originais e internacionais. Após leitura criteriosa das publicações, 4 artigos não foram utilizados devido aos critérios de exclusão. Assim, totalizaram-se 12 artigos científicos para a revisão. Resultados: A apendicite aguda demonstrou ser o maior exemplo de condição cirúrgica em que o cirurgião pediátrico pode realizar a videolaparoscopia e oferecer uma melhor evolução ao paciente em relação à técnica convencional. No entanto, notou-se que quanto menor a idade, maiores os riscos de prejuízos e não recomendação desse procedimento, especialmente em recém-nascidos. Conclusão: Em crianças, a videolaparoscopia é indicada com segurança para apendicectomia, retirada da vesícula, correção de fluxo gastroesofágico, testículo intra-abdominal (quando o testículo não desceu para a bolsa escrotal), cisto no ovário e alguns tipos de biópsias.
\end{abstract}

Palavras-chave: Videolaparoscopia; Cirurgia; Pediatria. 


\begin{abstract}
Introduction: laparoscopic surgery is one of the five main fields of progress in pediatric surgery in the last decade of the 20th century and has been a progressive advance that allowed the development of a large number of surgical procedures using minimally invasive technique. However, it is worth analyzing in a special way how this technique is developed in pediatric patients, since there are complications and applications for this population group, which needs careful evaluations when performing procedures. Objective: to analyze case reports, articles and studies that show the advantages and disadvantages of performing videolaparoscopy surgery in pediatric patients Methodology: This is a systematic literature review with studies published in the following databases: National Library of Medicine (PubMed MEDLINE), Scientific Electronic Library Online (Scielo), Cochrane Database of Systematic Reviews (CDSR), Google Scholar, Virtual Health Library (VHL) and EBSCO Information Services. Relevant sources inherent to the theme were analyzed, using as one of the main criteria the choice of current, original and international articles. After careful reading of the publications, 4 articles were not used due to the exclusion criteria. Thus, there were a total of 12 scientific articles for review. Results: Acute appendicitis proved to be the greatest example of a surgical condition in which the pediatric surgeon can perform videolaparoscopy and offer a better evolution to the patient compared to the conventional technique. However, it was noted that the younger the age, the greater the risk of damage and that this procedure is not recommended, especially in newborns. Conclusion: In children, videolaparoscopy is safely indicated for appendectomy, gallbladder removal, gastroesophageal flow correction, intra-abdominal testis (when the testicle has not descended into the scrotum), ovarian cyst and some types of biopsies.
\end{abstract}

Keywords: Videolaparoscopy; Surgery; Pediatrics.

\title{
Resumen
}

Introducción: la cirugía laparoscópica es uno de los cinco principales campos de avance de la cirugía pediátrica en la última década del siglo XX y ha supuesto un avance progresivo que permitió el desarrollo de un gran número de procedimientos quirúrgicos con técnica mínimamente invasiva. Sin embargo, vale la pena analizar de manera especial cómo se desarrolla esta técnica en pacientes pediátricos, ya que existen complicaciones y aplicaciones para este grupo de población, que necesita evaluaciones cuidadosas a la hora de realizar los procedimientos. Objetivo: analizar casos clínicos, artículos y estudios que muestren las ventajas y desventajas de realizar cirugía de videolaparoscopia en pacientes pediátricos Metodología: Se trata de una revisión sistemática de la literatura con estudios publicados en las siguientes bases de datos: National Library of Medicine (PubMed MEDLINE), Scientific Electronic Library Online (Scielo), Cochrane Database of Systematic Reviews (CDSR), Google Scholar, Virtual Health Library (BVS) y EBSCO Information Services. Se analizaron fuentes relevantes inherentes a la temática, utilizando como uno de los principales criterios la elección de artículos actuales, originales e internacionales. Tras una lectura atenta de las publicaciones, 4 artículos no se utilizaron debido a los criterios de exclusión. Así, hubo un total de 12 artículos científicos para revisión. Resultados: La apendicitis aguda resultó ser el mayor ejemplo de condición quirúrgica en la que el cirujano pediatra puede realizar una videolaparoscopia y ofrecer una mejor evolución al paciente en comparación con la técnica convencional. Sin embargo, se observó que a menor edad, mayor riesgo de daño y que este procedimiento no es recomendable, especialmente en recién nacidos. Conclusión: En niños, la videolaparoscopia está indicada con seguridad para apendicectomía, extirpación de la vesícula biliar, corrección del flujo gastroesofágico, testículo intraabdominal (cuando el testículo no ha descendido al escroto), quiste ovárico y algunos tipos de biopsias.

Palabras clave: Videolaparoscopia; Cirugía; Pediatría.

\section{Introdução}

A cirurgia laparoscópica é um dos cinco principais campos de progresso na cirurgia pediátrica na última década do século XX e tem sido um avanço progressivo o que permitiu o desenvolvimento de um grande número de procedimentos cirúrgicos por técnica minimamente invasiva. O desenvolvimento dessas técnicas também tem sido associado a uma curva lenta e longa de relatórios sobre a segurança, eficácia e custo-benefício de cirurgias laparoscópicas, sendo raras em crianças, e têm mostrado uma grande evolução em curto tempo. Essa evolução influenciou a maioria dos procedimentos pediátricos, mudando os paradigmas da prática da cirurgia em crianças, que comumente se beneficiaram dessas mudanças pelo uso de incisões menores, menos dor, menor tempo de internação e rápida incorporação às suas atividades usuais. Ou seja, a cirurgia videolaparoscópica ou laparoscopia tem se difundido como um método minimamente invasivo no tratamento de diversas patologias infantis, principalmente na remoção de órgãos inflamados (principalmente a remoção do apêndice para casos pediátricos (Uribe et al 2021). 
No entanto, cabe analisar de forma especial como essa técnica se desenvolve em pacientes pediátricos, visto que, há complicações e aplicações para esse grupo da população, que necessita de avaliações criteriosas quando se realiza procedimentos, seja sob questões anestésicas, seja sob o pré e pós operatório, bem como experiência do cirurgião e aperfeiçoamento das áreas cirúrgicas. Além disso, cabe ressaltar que uma das dificuldades nos estudos com crianças é a maior dificuldade de aprovação dos testes e do consentimento dos pais e da comissão de ética, inclusive foi observado em variados estudos a não permissão dos pais na publicação dos resultados. Por isso, muitos estudos acabam não sendo realizados nesses grupos atrasando o desenvolvimento de técnicas mais modernas e que podem melhorar a qualidade de vida desses infantes (Oliveira et al. 2020). Portanto, o objetivo dessa revisão sistemática de literatura é analisar relatos de caso, artigos e estudos que mostrem quais são as vantagens e as desvantagens da realização da cirurgia videolaparoscopia em pacientes pediátricos. Assim, analisando quais seriam as complicações e as aplicações mais favoráveis no desenvolvimento das técnicas cirúrgicas para esse grupo populacional.

\section{Metodologia}

Trata-se de uma pesquisa descritiva do tipo revisão sistemática da literatura. Para a elaboração da questão de pesquisa, utilizou-se a estratégia PICOT (Acrômio para Patient, Intervention, Comparation, Outcome and Time). $\mathrm{O}$ uso dessa estratégia para formular a questão de pesquisa na condução de métodos de revisão possibilita a identificação de palavras-chave, as quais auxiliam na localização de estudos primários relevantes nas bases de dados. Assim, a questão de pesquisa delimitada foi: quais são as aplicações da cirurgia videolaparoscópica em pacientes pediátricos?”. Dessa maneira, compreende-se que $\mathrm{P}=$ pacientes pediátricos; $\mathrm{I}=$ pacientes pediátricos que foram submetidos à cirurgia videolaparoscópica; $\mathrm{Co}=$ pacientes pediátricos que não foram submetidos à cirurgia videolaparoscópica; $\mathrm{T}$ = dois meses de coleta de dados.

A partir do estabelecimento das palavras-chave da pesquisa, foi realizado o cruzamento dos descritores, em português: "pediatria", "videolaparoscopia", "cirurgia", "indicações", "apendicitectomia", em inglês: "pediatrics", "videolaparoscopy", "surgery", "indications", "appendectomy" e em espanhol: "pediatría", "videolaparoscopia", "cirugía", "indicaciones", "apendicectomía" nas seguintes bases de dados: National Library of Medicine (PubMed MEDLINE), Scientific Electronic Library Online (Scielo), Cochrane Database of Systematic Reviews (CDSR), Google Scholar, Biblioteca Virtual em Saúde (BVS) e EBSCO Information Services. A pesquisa bibliográfica foi de cunho exploratório, partindo da identificação, da seleção e da avaliação de trabalhos e de artigos científicos considerados relevantes para dar suporte teórico para a classificação, a descrição e a análise dos resultados.

A busca foi realizada nos meses de outubro e novembro 2021. Foram considerados estudos publicados no período compreendido entre 2001 e 2021. A estratégia de seleção dos artigos seguiu as seguintes etapas: busca nas bases de dados selecionadas; leitura dos títulos de todos os artigos encontrados e exclusão daqueles que não abordavam o assunto; leitura crítica dos resumos dos artigos e leitura na íntegra dos artigos selecionados nas etapas anteriores. Analisou-se fontes relevantes inerentes ao tema, utilizando como um dos principais critérios a escolha de artigos atuais, originais e internacionais. Após leitura criteriosa das publicações, 4 artigos não foram utilizados devido aos critérios de exclusão. Assim, totalizaram-se 12 artigos científicos para a revisão sistemática da literatura, com os descritores apresentados acima. Como critérios de inclusão, foram considerados artigos originais, que abordassem o tema pesquisado e permitissem acesso integral ao conteúdo do estudo, no período de 2001 a 2021, em português, inglês e espanhol. O critério de exclusão foi imposto naqueles trabalhos que não estavam nesses idiomas, que não tinham passado por processo de Peer-View e que não se relacionassem com o tema, conforme elucidado pelo Diagrama 1. 
Diagrama 1: Etapas de seleção de artigos para esta pesquisa.
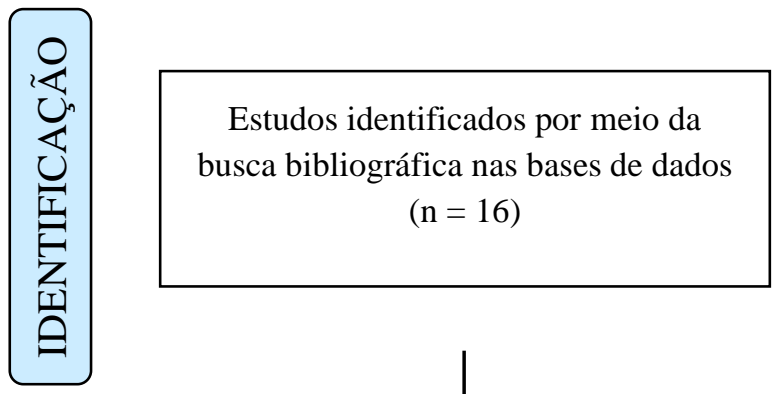

N. de relatos identificados em outras fontes $(n=0)$
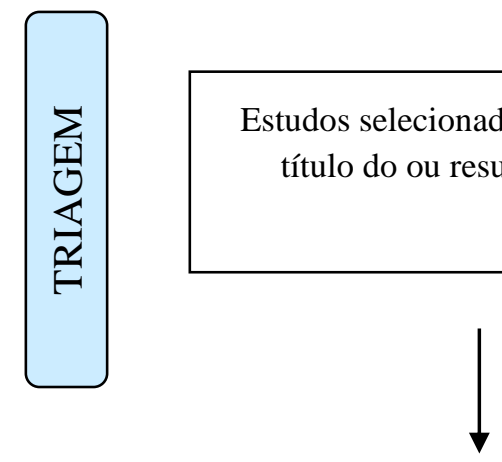

Estudos selecionados para verificação de critérios de inclusão $(\mathrm{n}=15)$
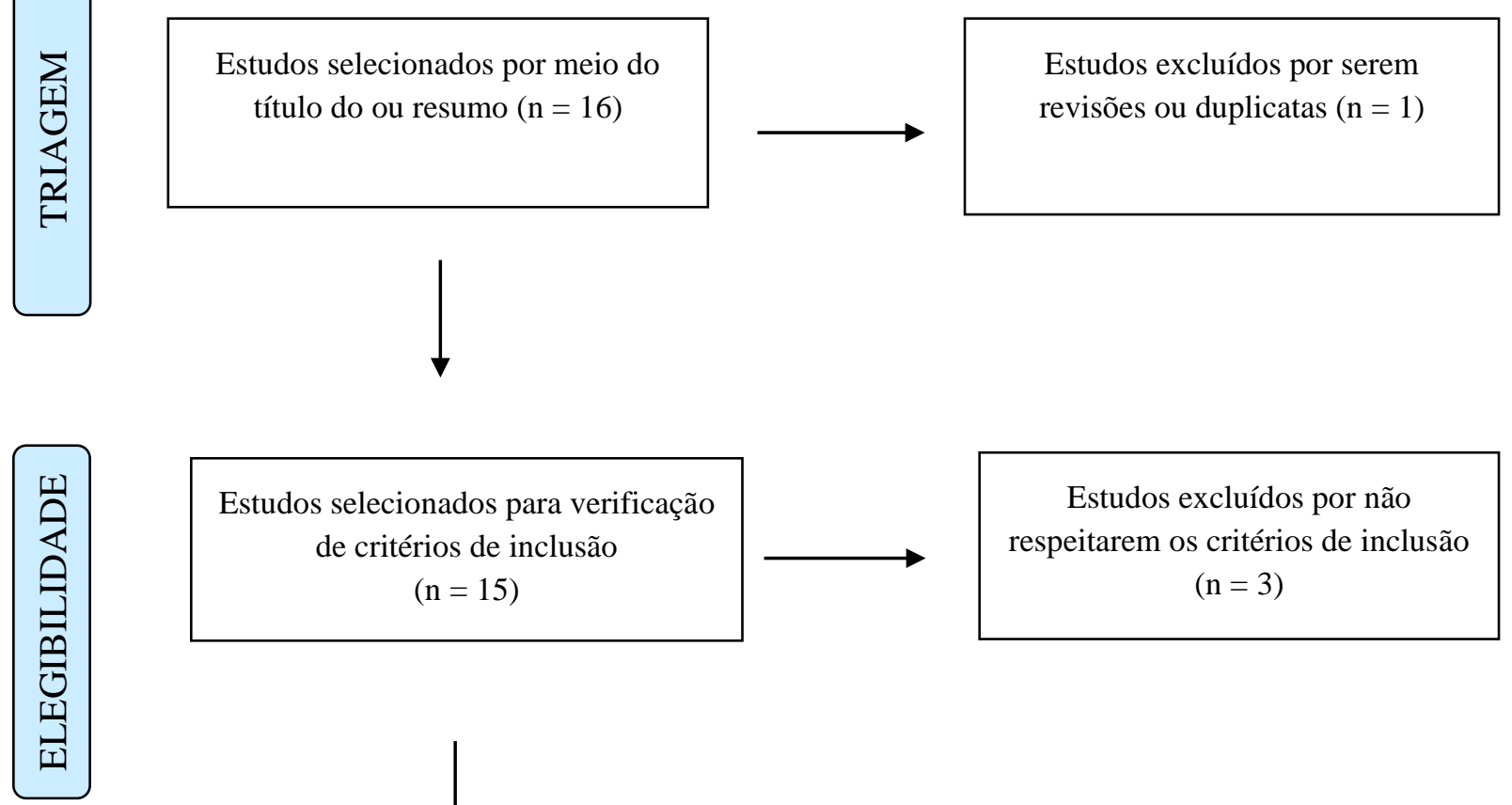

Estudos incluídos para a revisão de literatura $(n=12)$

Fonte: Autores.

\section{Resultados e Discussão}

O acesso laparoscópico é comumente escolhido em casos em que habitualmente se indicaria laparotomia, dependendo de fatores como previsão de incisões da laparoscopia menores ou mais estéticas, crianças obesas, adolescentes, dúvida diagnóstica, biópsias, disponibilidade do aparelho ou acessórios, indicações laparoscópicas consagradas como colecistectomia, refluxo gastroesofágico e outros. A fim de acoplar as evidências encontradas nesta pesquisa sistemático, realizou-se um quadro (Quadro 1) com os principais achados dos estudos. 
Quadro 1: Principais achados da pesquisa.

\begin{tabular}{|c|c|}
\hline AUTORES E ANOS & PRINCIPAIS ACHADOS \\
\hline Misauno et al. 2012 & $\begin{array}{l}\text { A cirurgia videolaparoscópica para o tratamento da apendicite em pacientes pediátricos tem sido usada de forma } \\
\text { frequente, mostrando-se como um procedimento com menos complicações e melhor qualidade de vida pós } \\
\text { operatória. Aliado a isso, a dexmedetomidina tem sido um anestésico que mostrou ótima reação em crianças. }\end{array}$ \\
\hline Esteves et al. 2001 & $\begin{array}{l}\text { A utilização do método laparoscópico mostrou-se como uma alternativa para uma melhora na recuperação e com } \\
\text { menor uso de remédios para aliviar a dor em crianças no pós operatório. }\end{array}$ \\
\hline Duarte et al. 2014 & $\begin{array}{l}\text { Em cirurgias para retirada de tumor de Wilms não se observou grandes diferenças quando se optou pela } \\
\text { laparoscopia em relação a laparotomia. }\end{array}$ \\
\hline Testa et al. 2020 & $\begin{array}{l}\text { A técnica de videolaparoscopia se mostrou como padrão ouro para o manejo de cistos benignos ovarianos em } \\
\text { pacientes pediátricos. No entanto, a técnica ainda não foi consolidada. }\end{array}$ \\
\hline Oliveira et al. 2020 & $\begin{array}{l}\text { A colescistectomia por meio da técnica de vídeo é considerado padrão ouro para cirurgias pediátricas. Nesse } \\
\text { sentido, ocorreram poucas complicações em pacientes maiores de } 5 \text { anos. }\end{array}$ \\
\hline Goyal et al. 2020 & $\begin{array}{l}\text { As aplicações e complicações da laparoscopia infantil se mostraram semelhantes em comparação com os adultos. } \\
\text { No entanto, os recém nascidos tendem a ter mais complicações cardíacas, no pneumoperitônio e com relação aos } \\
\text { anestésicos. }\end{array}$ \\
\hline Pinheiro et al. 2014 & A apendicectomia por incisão videolaparoscópica é uma aplicação difundida para casos pediátricos. \\
\hline Djiré et al. 2013 & $\begin{array}{l}\text { Ocorreu uma redução na incidência de escoliose pós-toracotomia, o que mostrou ser uma aplicação para pacientes } \\
\text { pediátricos na ligadura por videolaparoscopia. }\end{array}$ \\
\hline Jiménez-Uribe et al. 2021 & $\begin{array}{l}\text { A apendicectomia por videolaparoscopia ou por laparotomia obtiveram resultados semelhantes quanto as } \\
\text { complicações e aplicações, por isso se mostraram como técnicas favoráveis em pacientes pediátricos. }\end{array}$ \\
\hline Carvalho et al. 2013 & $\begin{array}{l}\text { A revisão de shunt feita por laparoscopia se mostrou uma aplicação positiva em pacientes pediátricos. No entanto, } \\
\text { em pacientes menores de } 8 \text { anos foram observadas maiores complicações. }\end{array}$ \\
\hline Bertot et al. 2015 & $\begin{array}{l}\text { A colescistectomia laparoscópica se mostrou uma aplicação viável para pacientes pediátricos. As complicações } \\
\text { mais observadas foram relacionadas ao risco cirúrgico e anestésicos, que são maiores em crianças. }\end{array}$ \\
\hline Oliveira, 2005 & $\begin{array}{l}\text { O aperfeiçoamento das técnicas instrumentais para crianças tem permitido que as cirurgias minimamente invasivas } \\
\text { sejam amplamente empregadas nos tratamentos pediátricos. }\end{array}$ \\
\hline
\end{tabular}

Fonte: Dados da pesquisa (2021).

A cirurgia laparoscópica permitiu realizar vários procedimentos abdominais convencionalmente efetuados por via aberta, sobretudo no tratamento do refluxo gastroesofágico, abdome agudo inflamatório, criptorquidia, obstrução por bridas, litíase biliar, tumores, afecções de ovário, esplenectomia, aganglionose, trauma e outros (Esteves et al., 2001). A morbidade foi baixa (1\%) e a mortalidade devido à laparoscopia foi nula. Houve conversão para laparotomia em somente 14 casos (2,3\%), sobretudo em trauma. As principais vantagens incluíram mínimos trauma cirúrgico, dor e íleo paralítico, pouco tempo de internação, quase ausência de hérnia incisional e melhor estética (Duarte et al., 2014).

De acordo com os resultados observados por Testa et al. (2020), a cirurgia videolaparóscopia é padrão ouro para diversas técnicas aplicadas na cirurgia pediátrica. Os achados mostraram que a maior parte das complicações são as observadas também em adultos como com relação aos anestésicos e ao pneumoperitônio. No entanto, notou-se que quanto menor a idade, maiores os riscos de prejuízos e não recomendação desse procedimento, especialmente em recém-nascidos. Entretanto, os tratamentos minimamente invasivos estão sendo amplamente utilizados nas aplicações pediátricas, principalmente quando ocorrem em estruturas hospitalares adequadas e especializadas (Oliveira et al., 2020).

Dessa forma, as melhorias no pós operatório, menor dor, menos uso de analgésicos, diminuição no tempo de repouso pós operatório, melhor cicatrização e uma estética positiva foram amplamente observadas nos estudos infantis que são semelhantes aos resultados encontrados nos adultos (Oliveira, 2005). No entanto, é importante que haja um aperfeiçoamento constante do profissional, das técnicas operatórias e dos instrumentais para que sejam adaptadas de forma positiva para um público diferente do adulto. Portanto, os estudos mostraram que a aplicação das cirurgias minimamente invasiva são positivas para a cirurgia pediátrica. Assim, torna-se possível afirmar pelos autores que o método deve ser amplamente difundido quando tomados os cuidados necessários que são especiais para crianças (Carvalho et al., 2013).

A apendicite aguda demonstrou ser o maior exemplo de condição cirúrgica em que o cirurgião pediátrico pode realizar a videolaparoscopia e oferecer uma melhor evolução ao paciente em relação à técnica convencional. Atualmente, Bertot et al. (2015) demonstraram que a resposta inflamatória sistêmica e os efeitos inflamatórios produzidos por trauma 
cirúrgico são menores quando a técnica utilizada é a videolaparoscopia, e embora ainda controverso, é útil para a evolução particular de pacientes. Além disso, Jiméenz-Uribe et al. (2021) alegam que modificar o procedimento, ou seja, converter uma cirurgia de acesso mínimo à cirurgia convencional por qualquer motivo, não deve ser considerado uma complicação, mas uma decisão estratégica, em que a forma de acesso é alterada para a solução adequada de um problema cirúrgico grave, tendo sempre como denominador comum a finalidade de evitar a iatrogenia no paciente (Tinoco et al., 2006).

Ademais, a técnica laparoscópica básica consiste em, estando a criança sob anestesia geral, realiza-se uma pequena incisão o intra-umbilical para colocação de um trocarte sob visão direta do peritônio, ou introduz-se uma agulha de Veress na cicatriz umbilical (Misauno et al. 2012). Pelo trocarte ou pela agulha é infundido CO2 na cavidade peritoneal com um insuflador automático. O pneumoperitônio expande-se até a pressão máxima de 8 a $10 \mathrm{mmHg}$, fixada previamente no insuflador, afasta a parede abdominal das vísceras e forma o espaço de trabalho na cavidade. O gás carbônico (CO2) não é inflamável com eletrocautério e é rapidamente difusível para o ar expirado pelos pulmões, e durante toda a operação, a criança é monitorizada com oxi-capnógrafo. Um trocarte de 4 ou $10 \mathrm{~mm}$ é introduzido no umbigo ou em outro ponto de melhor visão da área a ser operada (Goyal et al. 2020).

Por este trocarte, uma óptica conectada a uma microcâmera e uma fonte de luz permitem iluminar e visibilizar a cavidade, mostrada em uma televisão ou monitor de vídeo. Sob visão interna da câmera, outros trocartes finos são passados pela parede por perfurações de 3 a $5 \mathrm{~mm}$ conforme a operação proposta. A óptica aumenta a visão até 16 ou 20 vezes, ampliando-se detalhes mínimos no intra-operatório (Djiré et al. 2013). Nos orifícios são introduzidos os instrumentais finos de trabalho (pinças, tesouras, porta-agulhas, aspirador, etc.). Todos os procedimentos, inclusive suturas e anastomoses, podem ser realizados. A retirada de órgãos se faz pelo umbigo ou pequenas inguinotomias, podendo também se fragmentar os órgãos ou tumores dentro de sacos plásticos introduzidos, removendo-se os fragmentos pela boca do saco exteriorizado no umbigo (Pinheiro et al. 2014).

\section{Conclusão}

Em crianças, a videolaparoscopia é indicada com segurança para apendicectomia, retirada da vesícula, correção de fluxo gastroesofágico, testículo intra-abdominal (quando o testículo não desceu para a bolsa escrotal), cisto no ovário e alguns tipos de biópsias. Conclui-se que a videolaparoscopia parece ser um grande avanço da cirurgia pediátrica moderna, permitindo tratar de modo menos invasivo e com muita segurança grande parte das afecções abdominais em crianças de todas idades, demonstrando-se inúmeras vantagens sobre as laparotomias, quando bem indicadas e realizadas dentro dos princípios cirúrgicos e anestésicos corretos.

\section{Referências}

Bertot, J. G., et al. (2015). Characterization of surgically treated patients by means of laparoscopic cholecystectomy in a pediatric hospital. MediSan, 19 (4), $468-476$.

Carvalho, F. O., et al. (2013). Laparoscopic assisted ventriculoperitoneal shunt revisions as an option for pediatric patients with previous intraabdominal complications. Arq Neuropsiquiatr., 72 (4), 307-311.

Djiré, M. K., et al. (2013). Caracterización de la cirugía videolaparoscópica de urgência em la infância. Rev Colom Cir., 32 (1), 1-8.

Duarte, R. J., et al. (2014). Wilms Tumor: A Retrospective Study of 32 Patients Using Videolaparoscopic and Open Approaches. Pediatric Urology, 5 (8), 1-7.

Esteves, E., et al. (2001). Aplicações da cirurgia laparoscópica em crianças. J. Pediatr., 77 (5), 407-413.

Goyal, R., et al. (2020). Anestesia para cirurgia videolaparoscópica pediátrica. Anaesthesia, 28 (1), 1-6.

Jiménez-Uribe, A. M., et al. (2021). Cirugía transumbilical asistida versus multipuerto en pacientes pediátricos com diagnóstico de apendicitis aguda. Rev Colom Cir., 36 (8), 481-486. 
Research, Society and Development, v. 10, n. 15, e408101523165, 2021

(CC BY 4.0) | ISSN 2525-3409 | DOI: http://dx.doi.org/10.33448/rsd-v10i15.23165

Misauno, M. A., et al. (2012). Laparoscopic pediatric surgery: a potentil for paradigm shift in developing countries. African Journal of Paediatric Surgery, 9 (2), 140-143.

Oliveira, C. R. D. (2005). Anestesia para cirurgia videolaparoscópica. Rev Bras Videocir., 3 (1), 32-43.

Oliveira, P. A., et al. (2020). Colelitíase na infância e adolescência: abordagem diagnóstica e tratamento. Revista Médica de Minas Gerais, 9 (5), $22-26$.

Pinheiro, R. N., et al. (2014). Videoapendicectomia por incisão única com material de videolaparoscopia concenvional. Arq Bras Cir Dig., 27 (1), $34-37$.

Testa, C. P., et al. (2020). Video laparoscopic approach of a giant ovarian cyst in a pediatric patient: Case Report. Revista Médica de Minas Gerais, 9 (49), 18.

Tinoco, R. C., et al. (2006). Ligadura Videolaparoscópica do Canal Arterial Persistente: Experiência de Cinquenta e Um Casos. Rev Bras Videocir., 4 (4), 166170. 